\author{
MATTAUCH, LINUS ${ }^{1}$ \\ SIEGMEIER, $J A N^{2}$ \\ FUNKE, FRANZISKA ${ }^{3}$
}

\title{
Wirtschaftswachstum aufgeben?
}

Zur Struktur wachstumskritischer Argumente

\section{POST-PRINT: Im Erscheinen in Zeitschrift für Wirtschafts- und Unternehmensethik (2019). 20/1}

Der Beitrag prüft Argumente dafür, dass weiteres Wirtschaftswachstum mit Klimaschutz nicht zu vereinbaren und angesichts negativer gesellschaftlicher Auswirkungen nicht erstrebenswert sei. Von einer gegenwärtig inadäquaten Regulierung kann jedoch nicht auf die Unmöglichkeit einer adäquaten in der Zukunft geschlossen werden. Selbst wenn gegenwärtiges Wachstum nicht förderlich für manche gesellschaftlichen Ziele ist, folgt nicht, dass man diese durch weniger Wachstum besser erreicht.

Schlagwörter: Postwachstum, Nachhaltiges Wirtschaftswachstum, Klimawandel, Wohlfahrtstheorie

\section{Giving up on Growth? On the Validity of Post-Growth Arguments}

We analyse arguments on the supposed incompatibility of economic growth with climate change mitigation and human welfare. Current inadequacies in the regulation of environmental issues, however, do not imply that pathways of sustainable growth are categorically unattainable. Further, while economic growth as such might not contribute to certain societal goals, it does not follow that those goals can be achieved in its absence.

\footnotetext{
${ }^{1}$ Dr. Linus Mattauch, Institute for New Economic Thinking at the Oxford Martin School and Environmental Change Institute, School of Geography and the Environment, Universität Oxford, Eagle House, Walton Well Road, Oxford Ox2 6ED, United Kingdom, E-Mail: linus.mattauch@inet.ox.ac.uk

Forschungsschwerpunkte: Klimaökonomik, Finanzwissenschaft, Wachstumstheorien, Philosophie der Volkswirtschaftslehre

${ }^{2}$ Dr. Jan Siegmeier, Mercator Research Institute on Global Commons and Climate Change Berlin. E-Mail: siegmeier@mcc-berlin.net

${ }^{3}$ Franziska Funke, London School of Economics and Political Science. E-Mail: f.funke@1se.ac.uk
} 
Keywords: Post-growth economics, Degrowth, Sustainable Growth, Climate Change, Welfare theory 


\section{Einleitung}

Angesichts des Klimawandels und weiterer globaler Umweltprobleme, Finanz- und Staatsschuldenkrisen und einer als zu stressig empfundene Arbeitswelt nimmt die Kritik an einem wachstumsorientierten Wirtschaftssystem zu. Das gesellschaftliche Phänomen der „Postwachstumsbewegung“ in Deutschland und anderen Industrieländern wird flankiert von einer breit rezipierten wachstumskritischen Literatur (Jackson, 2017; Kallis, 2011; Latouche, 2015; Paech, 2013) und "Degrowth"-Konferenzen. Häufige Forderungen sind eine Abkehr von Wachstum als gesellschaftlichem Ziel und dem Bruttoinlandsprodukt als zentralem Wohlfahrtsindikator, die radikale Veränderung von Konsum- und Produktionsmustern und ein Schrumpfen der Wirtschaft zur Vermeidung von Umweltkatastrophen, sowie ein gesellschaftlicher Dialog über Wohlstand und Glück. An der tradierten Volkswirtschaftslehre wird beanstandet, dass ihre konsumfokussierten Standardmodelle negative Folgen des Wachstums nicht abbilden.

Wachstumskritische AutorInnen treffen mit ihrer Intuition, die Vorrangstellung von Wirtschaftswachstum und Konsum zu hinterfragen, den Zeitgeist. Sie fordern zu Recht, die ökologischen, sozialen und psychischen Dimensionen des Wirtschaftens ins Zentrum wirtschaftspolitischer Debatten zu stellen (Raworth, 2017). Allerdings bleibt ihre Argumentation teils lückenhaft und undurchsichtig: wird das gesamte Spektrum an wirtschaftspolitischen Möglichkeiten zur Vermeidung ökonomischer Krisen sowie des Klimawandels und zur Verbesserung der Lebensqualität erfasst? Und selbst wenn Wachstum um seiner selbst willen kein sinnvolles gesellschaftliches Ziel darstellt, ist die umgekehrte Fokussierung auf „Nicht-Wachstum“ oder Schrumpfung tatsächlich geeignet oder sogar zwingend erforderlich, um Ziele der Nachhaltigkeit und des guten Lebens zu erreichen?

Ziel des vorliegenden Beitrags ist es, zwei zentrale Argumente, die von wachstumskritischen Stimmen oftmals vorgebracht werden, kritisch zu bewerten. Dazu untersuchen wir erstens die Fragestellung ,Müssen wir Wachstum aufgeben?“ beispielhaft anhand der Vereinbarkeit von weiterem Wirtschaftswachstum mit Klimaschutzzielen. Wir zeigen auf, dass häufig von der bisher fehlgeschlagenen Entkopplung von Wachstum und Emissionen voreilig auf deren prinzipielle Unmöglichkeit geschlossen wird. Insbesondere werden sektorspezifische technische Möglichkeiten zur Emissionsreduktion, Politikinstrumente um diese auszuschöpfen sowie deren politische Machbarkeit nicht sorgfältig unterschieden und analysiert. In der Konsequenz wird die Wirkung klimapolitischer Maßnahmen übersehen oder bezweifelt, die durchaus anspruchsvoll, aber potentiell ausreichend wären, etwa bei Strom und Wärme, Transport und Ernährung (IPCC, 2014). Stattdessen wird eine Wachstumsrücknahme gefordert, die ungleich schwieriger zu realisieren wäre und womöglich mit erheblichen sozialen Kosten einherginge.Dieser Beitrag stellt nur die Vereinbarkeit von Wirtschaftswachstum mit Klimaschutz im Detail vor, weil es unter den globalen Umweltproblemen eine zentrale Stellung einnimmt. Ähnliches gilt für andere häufig diskutierte natürliche Wachstumsgrenzen, wie Biodiversitätsverlust, Überfischung oder Rohstoffknappheit.

Die zweite Fragestellung, „Wollen wir Wachstum aufgeben?“, adressiert die psychologische Bedeutung von Konsum und Wachstum für das Glück der Bevölkerung 
sowie die normative Begründung für Wirtschaftswachstum. Es zeigt sich, dass diese beiden Aspekte häufig nicht sorgfältig unterschieden werden: WachstumskritikerInnen verweisen zurecht auf empirische Analysen der Glücksforschung und Sozialpsychologie, die darauf hindeuten, dass Wachstum in seiner Wirkung auf das Glück der Menschen überschätzt wurde. Ein positiver Zusammenhang im langfristigen Ländervergleich ist möglich, aber nur schwach ausgeprägt (Easterlin et al., 2010; Kahneman und Deaton, 2010; Layard, 2011). Dabei übersehen sie aber die entscheidende normative Frage, ob denn Glück ein geeigneteres Wohlfahrtskriterium darstellen kann als die Erfüllung individueller Präferenzen (Hausman, 2012), welche die neoklassische Ökonomik ins Zentrum stellt.

Der vorliegende Beitrag bringt erstmals eine argumentative Kritik tradierter wachstumskritischer Positionen zusammen, die sowohl die Wachstumsmöglichkeiten angesichts planetarer Grenzen betreffen als auch die Erwünschtheit von Wirtschaftswachstum. Dies erscheint nötig, weil beide Arten von Wachstumskritik bisweilen unzulässig vermischt werden: Die Frage, ob weiteres Wirtschafswachstum, jedenfalls in reichen Ländern, überhaupt erstrebenswert sei, wäre selbst dann noch von zentraler Bedeutung, wenn die Möglichkeit weiteren Wachstum gezeigt wäre. Verwandte Beiträge verteidigen die Umweltökonomik trotz ihrer Schwachstellen gegen Wachstumskritik (Hepburn und Bowen, 2013; Jakob und Edenhofer, 2014; van den Bergh, 2017). Sie arbeiten aber nicht heraus, dass die These, dass Wirtschaftswachstum zugunsten des Klimaschutzes aufgegeben werden müsse, einen Fehlschluss von einer spezifischen, nicht-optimalen Vergangenheit auf die Unmöglichkeit einer besseren zukünftigen Regulierung von Umweltproblemen darstellt. Andere AutorInnen betrachten die wirtschaftspolitischen Empfehlungen, die sich ergeben, wenn man Glückssteigerung statt Präferenzerfüllung als normativen Standpunkt annimmt, um Konsum zu bewerten (Layard, 2011). Sie vergleichen aber keine normativen Positionen zum individuellen Wohlergehen wie etwa Fleurbaey und Blanchet (2013).

Wir beschränken uns auf eine argumentative Analyse zweier im wachstumskritischen Diskurs vorgebrachter Argumente und fassen die Vielfalt wachstumskritischer AutorInnen und ihrer Positionen nur zur Einordnung knapp zusammen (vgl. Schmelzer, 2015; Leipprand und aus dem Moore, 2012). Die in Abschnitt 2 analysierten Argumente werden in ihrer Reinform von kaum einem/r AutorIn explizit vertreten, jedoch oftmals im öffentlichen Diskurs so vorgebracht. Wir zeigen auf, was tatsächlich aus diesen Behauptungen folgt, und stellen fest, dass sich auf ihrer Basis kein überzeugendes Argument für eine Wachstumsrücknahme ergibt. Dabei betrachten wir auch deshalb bewusst zugespitzte Argumente für das Aufgeben von Wachstum, um Einsichten über die Berechtigung darüberhinausgehender kritischer Sichtweisen auf Wirtschaftswachstum abzuleiten. Diese werden in Abschnitt 3 besprochen; Abschnitt 4 schließt mit Implikationen für die Kommunikation volkswirtschaftlicher Forschung. 


\section{Überblick über die Wachstumskritik}

Wachstumskritik kann bis zum Beginn der industriellen Revolution zurückverfolgt werden, als Malthus angesichts einer rasant wachsenden britischen Bevölkerung Grenzen des Wachstums durch knappe Flächen und Lebensmittel gegeben sah (Malthus, 1798). Technologischer Fortschritt und Ressourcenreichtum ließen seine pessimistischen Ansichten lange in den Hintergrund treten. Mit der vom Club of Rome in Auftrag gegebenen Studie „Limits to growth“ (Meadows et al., 1972) wurde die Frage nach der Endlichkeit des Wirtschaftswachstums im 20. Jahrhundert erneut Gegenstand breiter gesellschaftlicher Debatten, nun im Kontext der prognostizierten Knappheit fossiler Rohstoffe. Neben der demographischen Entwicklung, Ressourcenknappheit und Klimaschutz wurden mit dem Aufkeimen der Glücksforschung auch psychologische und soziale Grenzen des Wachstums diagnostiziert (Scitovsky, 1976).

In diesem Beitrag unterscheiden wir eine weite und eine enge Definition der Wachstumskritik. Wirtschaftswachstum wird hierbei als Wachstum des Bruttoinlandsprodukts definiert. In ihrer weiten Definition lässt sich Wachstumskritik als Kritik an einem Wachstumsparadigma verstehen, das ein Wachstum des Bruttoinlandsprodukts mit einer automatischen Steigerung der gesellschaftlichen Wohlfahrt gleichsetzt. Während die Wachstumskritik, weit gefasst, somit Wachstum als politisches Ziel hinterfragt, formuliert die Wachstumskritik in ihrer engen Form dezidiert „Kein-Wachstum“ als Lösungsmöglichkeit für gesellschaftliche Probleme und führt Argumente und Ansätze für eine induzierte Stagnation oder Schrumpfung der Wirtschaft an.

Ein Beispiel für Wachstumskritik nur im weiteren Sinn ist die „A-growth“-Strategie, die fordert, dass bei der Verwirklichung sozial- und wirtschaftspolitischer Ziele das Erreichen von Wachstum nur eine untergeordnete Rolle spielen sollte (van den Bergh, 2017; Jakob und Edenhofer, 2014; Raworth, 2017). Dieser und ähnliche Ansätze gehen mit einer Kritik am Bruttoinlandsprodukt als dominantem Wohlfahrtsindikator einher, welche von den Wirtschaftswissenschaften breit akzeptiert wird (Fleurbaey und Blanchet, 2013; Stiglitz et al., 2009) und der wir uns anschließen. Politisch ist die Abkehr von „Wachstum als Ziel“ jedoch durchaus kontrovers: Die Enquete-Kommission „Wachstum, Wohlstand, Lebensqualität" beleuchtet in ihrem Schlussbericht den gesellschaftlichen Stellenwert des Wirtschaftswachstums durchaus kritisch und zeigt verschiedene Handlungsalternativen in der Wohlfahrtsmessung auf (Deutscher Bundestag, 2013). Viele AkteurInnen und Institutionen setzen sich jedoch weiterhin explizit das Ziel, Wirtschaftswachstum zu schaffen, ohne Wachstum als Mittel zum Erreichen sozialpolitischer Ziele wie Verteilungsgerechtigkeit, Armutsbewältigung oder Bildung ausreichend zu hinterfragen.

Wachstumskritik in ihrer engen Definition wird begrifflich meist mit den Begriffen „Degrowth“ und Postwachstum assoziiert. Schmelzer (2015) identifiziert in einer Übersicht fünf dominante Strömungen in der gegenwärtigen deutschen Postwachstumsbewegung: sozialreformerisch, konservativ, suffizienzorientiert, kapitalismuskritisch und feministisch. 
Die Ansatzpunkte wachstumskritischer AutorInnen sind dabei vielfältig. Sozialreformerische Ansätze stellen die Auflösung der Wachstumsabhängigkeit gegenwärtiger Institutionen ins Zentrum (Seidl und Zahrnt, 2010). Konservative Wachstumskritik betont die unmittelbaren ökologischen und sozialen Folgen des Wachstums (Miegel, 2010), suffizienzorientierte Ansätze (Paech 2013) schließen zudem die Möglichkeit einer Entkopplung von Ressourcenverbrauch und Wachstum aus. Kapitalismuskritische (Schmelzer und Passadakis, 2011) und feministische Ansätze (Bennholdt-Thomsen, 2010) warnen vor der Krisenanfälligkeit auf Wachstum ausgerichteter Wirtschaftssysteme und verweisen auf Ausbeutungsverhältnisse gegenüber dem globalen Süden und nicht monetär erfassbarer Subsistenzwirtschaft wie der Hausarbeit. Auch in ihren politischen Forderungen unterscheiden sich wachstumskritische AutorInnen. Die Lösungsvorschläge reichen von der Veränderung bestehender Institutionen und Abkehr vom politischen Wachstumsparadigma (Seidl und Zahrnt, 2010) über die Förderung neuer Formen kooperativen Wirtschaftens (Loske, 2014) bis zur subsistenzorientierten Regionalwirtschaft und Arbeitszeitverkürzung (Paech, 2013). In diesem Zusammenhang wird unter anderem auch eine Verringerung der Distanz zwischen Produktion und Konsum und die Regionalisierung von Märkten und Währungssystemen vorgeschlagen (Paech, 2013). Weiterhin gehen die Vorschläge häufig mit der politischen Forderung nach gesamtgesellschaftlichen Transformationen und Wertewandel einher. Wachstumskritische Ansätze, die sich auf den französischen Vordenker der „Décroissance“ Serge Latouche beziehen, verstehen ihre Forderungen als „reformatorisches Programm“ gesamtgesellschaftlicher Veränderungen und dezidiert nicht als „Wachstumsrücknahme um der Wachstumsrücknahme willen“ (Latouche, 2015, S. 24). So fordert Latouche (2015) die Abkehr von Konsumorientierung und die DeÖkonomisierung vieler Lebensbereiche zur Erreichung eines ,,besseren Lebens“.

In diesem Beitrag beschäftigen wir uns mit Wachstumskritik nach der engen Definition, nämlich der expliziten Forderung nach „Degrowth“ zur Lösung gesellschaftlicher Probleme, exemplarisch anhand zweier weit verbreiteter wachstumskritischer Gedankengänge wie sie etwa bei Paech $(2009,2013)$ und Jackson (2017) erkennbar sind.

Erstens beinhaltet diese Position das Argument, dass eine absolute Entkopplung des Wirtschaftswachstums vom Ressourcen- und Naturverbrauch durch technologischen Fortschritt nicht möglich sei. Dieser Pessimismus gründet auf einer grundsätzlichen Skepsis hinsichtlich der Substituierbarkeit endlicher Ressourcen, deren Knappheit sich in einem absehbaren Horizont zu einem „Peak Everything“ verdichten werde (Paech, 2009, Heinberg, 2007). Als zweites Merkmal der zu betrachtenden Degrowth-Argumentation identifizieren wir die auf Ergebnissen der Glücksforschung basierende Aussage, dass Wirtschaftswachstum das individuelle Glück nicht mehrt, sodass es in der Entschleunigung abseits von materiellem Konsum zu suchen sei. 


\section{Eine Analyse wachstumskritischer Argumente}

Zunächst behandeln wir die Frage, ob weiteres Wirtschaftswachstum mit natürlichen Grenzen vereinbar ist. Die drängenden globalen Umweltprobleme der Gegenwart bestehen selbstverständlich nicht nur in gefährlichem Klimawandel, sondern überdies in Artensterben bzw. Biodiversitätsverlust; globaler Landnutzung samt Entwaldung; Störung der Phosphor- und Stickstoffkreisläufe; Beeinträchtigung des Lebensraumes Ozean (Versäuerung/Überfischung) sowie der Ozonschicht (Steffen et al. 2015). Wir beschränken uns in der folgenden argumentationstheoretischen Untersuchung auf das Klimaproblem und zwar aus drei Gründen: Erstens ist das Klimaproblem am besten verstanden und quantifiziert, daher die Argumentationsstruktur daran am anschaulichsten darzustellen. Zweitens hängen die Fragen der globalen Landnutzung, samt Artensterben und Entwaldung, mit dem Klimaproblem zusammen und zwar über den $\mathrm{CO}_{2}$-Kreislauf und die Verwendung von Biomasse als Lösungsstrategie zur Vermeidung des Klimawandels. Drittens gilt für vom Klimaproblem und der Nutzung fossiler Ressourcen ${ }^{4}$ unabhängige Umweltprobleme, wie etwa der Überfischung und Verschmutzung der Ozeane durch Plastikmüll, dass es weit weniger plausibel ist als für den Klimaschutz, dass eine adäquate Lösung Auswirkungen auf die Gesamtwirtschaft hat. Stattdessen ist die gezielte Regulierung dieser Umweltprobleme anzustreben.

Bisweilen berufen sich manche wachstumskritischen Stimmen auf ein thermodynamisches Argument (Georgescu-Roegen 1971), demnach eine vollständige Entkopplung physischer Produktion vom Verbrauch natürlicher Ressourcen unmöglich ist. Dieser Einwand wird jedoch erst auf einem viel längeren Zeithorizont relevant als Argumente bezüglich der Unvereinbarkeit von Klimawandel und Wirtschaftswachstum, bei der es zunächst um die Substitution $\mathrm{CO}_{2}$-intensiver durch $\mathrm{CO}_{2}$-arme Technologien in den nächsten Jahrzehnten geht. Letzteren erachten wir als den politikrelevanten Zeitraum, auf den wir uns hier beschränken. ${ }^{5}$

${ }^{4}$ Mit dem Klimaproblem zusammen fällt die Frage, ob ein möglicher Mangel an Rohstoffen, das Wirtschaftswachstum bremsen könnte. Denn ein Mangel an fossilen Brennstoffen besteht, relativ zum $2^{\circ} \mathrm{C}$-Kohlenstoffbudget, nicht (Jakob und Hilaire 2015). Zudem kann ein möglicher Mangel an Mineralien voraussichtlich umgangen werden, wenn hinreichend viel $\mathrm{CO}_{2}$-neutrale Energie zur Gewinnung und Wiederverwertung von Mineralien bereitgestellt werden kann (Northey et al. 2014).

${ }^{5}$ In einem isolierten System bleibt zwar die Gesamtmenge an Energie konstant, ein zunehmender Teil dieser Energie ist aber aufgrund stetig zunehmender Entropie nicht mehr zur Verrichtung von Arbeit verfügbar. Allerdings ist die Erde aufgrund der eintreffenden Sonnenstrahlung kein isoliertes System - grob gesprochen kann die Menschheit auf die Dauer nur nicht mehr Energie verbrauchen (z.B. aus endlichen fossilen Vorräten, und inklusive des Aufwands für vollständiges Ressourcenrecycling), als sie Sonnenenergie „ernten“ kann (weitere ökologisch bedingte Beschränkungen, etwa bei Flächenverbrauch und „Biomasse-Durchsatz“, sind möglich). Heutige Windkraft- und Solaranlagen erzeugen jedoch über ihren Lebenszyklus hinweg deutlich mehr Energie als sie inklusive ihrer Herstellung verbrauchen (Sanden und Arvesen 2014, Louwen et al. 2016) und eröffnen so einen prinzipiell möglichen Ausweg, den Georgescu-Roegen (1986) noch als ,prometheisch“ abgetan hatte. 


\section{Müssen wir Wachstum für ambitionierten Klimaschutz aufgeben?}

Zusammenfassend lässt sich das im wachstumskritischen Diskurs vorgebrachte Argument, dass die Wirtschaft aus Gründen des Klimaschutzes stagnieren oder schrumpfen müsse, wie folgt darstellen:

1. Prämisse: Der Klimawandel ist menschengemacht und bedroht die natürlichen Lebensgrundlagen. Also müssen wir unser Wirtschaftssystem „dekarbonisieren“, d.h. die Gesamtmenge der Treibhausgasemissionen reduzieren.

2. Prämisse: Die nötige Dekarbonisierung ist bei weiterem Wirtschaftswachstum nicht zu schaffen.

Schlussfolgerung: Weiteres Wirtschaftswachstum muss verhindert werden.

Der Ausgangspunkt der ersten Prämisse entspricht dem naturwissenschaftlichen Konsens. Wie stark das Ausmaß und die Geschwindigkeit dieser Dekarbonisierung sein müssen, hängt dabei jedoch davon ab, welche Schäden und Risiken die Gesellschaft zu akzeptieren bereit ist. Die zweite Prämisse hingegen wird in mehrfacher Hinsicht nicht ausreichend begründet, da historisches Versagen und prinzipielle Unmöglichkeit verwechselt und technische und institutionelle Aspekte vermischt werden. Sie gilt selbst für ambitionierten Klimaschutz nur unter fragwürdigen Zusatzannahmen und verstellt den Blick auf die dringend nötige und anspruchsvolle Verbesserung gezielter Dekarbonisierungsinstrumente. Die Schlussfolgerung ist zudem nur dann ohne weiteres zulässig, wenn mögliche Konflikte und nötige Abwägungen mit anderen gesellschaftlichen Zielen ignoriert werden. Mit anderen Worten: Eine Schrumpfung der Wirtschaft wäre zwar wahrscheinlich hinreichend zur Erreichung ambitionierter Klimaziele - um zu zeigen, dass sie auch notwendig ist, müsste man jedoch zunächst zeigen, dass konventionelle Klimapolitik dieses nicht mehr erreichen kann. Dies analysieren wir nun im Einzelnen.

Die erste Prämisse des obigen Arguments ist plausibel: Es herrscht wissenschaftlicher Konsens darüber, dass anthropogene Emissionen für den Klimawandel verantwortlich sind und eine Erderwärmung von mehr als $2{ }^{\circ} \mathrm{C}$ über das vorindustrielle Niveau eine Bedrohung der Lebensgrundlagen jetziger und zukünftiger Generationen darstellt, beispielsweise durch Ernteverluste, Desertifikation und eine Erhöhung des Meeresspiegels (IPCC, 2014). Um das Ziel der Begrenzung auf $2{ }^{\circ} \mathrm{C}$ mit einer Wahrscheinlichkeit von wenigstens $66 \% \mathrm{zu}$ erreichen, dürfen nach Schätzungen des Weltklimarats die globalen $\mathrm{CO}_{2}$-Emissionen zwischen 2012 und 2100 die Gesamtmenge von $1000 \mathrm{GtCO}_{2}$ nicht überschreiten. Somit müssen in diesem Jahrhundert global null Netto-Emissionen erreicht werden. Dafür ist es erforderlich, die globalen jährlichen Emissionen beispielsweise bis 2050 schon um $40 \%$ bis $70 \%$ gegenüber $2010 \mathrm{zu}$ reduzieren (IPCC, 2014).

In ihrer Kritik an der konventionellen Klimapolitik verweisen wachstumskritische Stimmen darauf, dass vergangene Effizienzbestrebungen keine signifikante Dekarbonisierung der Wirtschaft herbeiführen konnten (Jackson, 2017). Zwar 
verzeichneten die OECD-Staaten zwischen 2007 und 2016 einen Rückgang der $\mathrm{CO}_{2^{-}}$ Emissionen um 8\% (IEA, 2016), dies bleibt jedoch weit hinter den Dekarbonisierungsleistungen zurück, die langfristig für die Einhaltung der Klimaziele notwendig wären. Währenddessen steigen die Emissionen in Entwicklungs- und Schwellenländern weiter. Global konnte zwar die $\mathrm{CO}_{2}$-Intensität reduziert werden, der absolute $\mathrm{CO}_{2}$-Ausstoß der wachsenden Weltwirtschaft ist jedoch weiterhin angestiegen (IPCC, 2014). Daraus leitet die Wachstumskritik ihre zweite Prämisse für die Notwendigkeit der Schrumpfung ab: Die notwendige Dekarbonisierung der Wirtschaft ist bei weiterem Wachstum nicht zu schaffen.

Diese Begründung der zweiten Prämisse ist jedoch nicht ausreichend, da sie sich auf vergangene Dekarbonisierungspfade stützt. Aus diesen folgt aber nicht, dass eine Dekarbonisierung der Weltwirtschaft bei anhaltendem Wirtschaftswachstums auch in Zukunft unmöglich sein wird. Um die argumentativen Fehler klar herauszuarbeiten, bietet die in der Volkswirtschaft gängige Unterscheidung zwischen Analysen des Erstbesten und Analysen des Zweitbesten (Lipsey und Lancaster, 1956) einen sinnvollen Rahmen. In einer Analyse des Erstbesten wird im Allgemeinen nur ein einziges Marktversagen untersucht, welches so durch staatliche Regulierung korrigiert werden kann, dass das gesellschaftliche Optimum erreichbar ist. In einer Analyse des Zweitbesten ist das nicht der Fall. Es liegen mehrere Marktversagen vor und es wird davon ausgegangen, dass mindestens eines nicht korrigiert werden kann, aber weitere Marktversagen optimal reguliert werden sollen (Lipsey and Lancaster, 1956; Perman et al. 2003). Angewendet auf die Klimapolitik heißt das: In einer Analyse des Erstbesten ist die negative Klimaexternalität die einzig vorhandene Restriktion bzw. wirtschaftliche Ineffizienz. Also werden allein technologische Vermeidungsoptionen und ihre Kosten evaluiert. Im Gegensatz dazu untersucht eine Analyse des Zweitbesten die Ausschöpfung jener technologischen Potentiale unter Berücksichtigung weiterer institutioneller Restriktionen und Ineffizienzen. ${ }^{6}$

Die von WachstumskritikerInnen behauptete prinzipielle Unvereinbarkeit von Emissionsreduktionen und Wachstum auch in der Zukunft ist entweder eine Aussage über das Erstbeste im Sinne fehlender technologischer Potentiale: Diese aus bisher unbefriedigenden Resultaten abzuleiten vernachlässigt aber die Möglichkeit, dass die Vergangenheit nur eine „Zweitbeste“ war, in der ungeeignete oder fehlende politische Maßnahmen verhinderten, dass möglicherweise vorhandene Dekarbonisierungspotentiale ausgeschöpft werden konnten (Helm, 2010). Oder sie ist eine Aussage über das Zweitbeste im Sinne einer Unmöglichkeit, klimapolitisch relevante Institutionen und

${ }^{6}$ Ein prominentes Beispiel für eine Analyse des Zweitbesten aus klimapolitischen Debatten ist das Argument, dass Klimaschutz „Ko-Benefits“ hätte, z.B. die Verbesserung lokaler Luftqualität. Dies ist nur dann stimmig, wenn es als zweitbeste Analyse verstanden wird, d.h. wenn vor der Durchsetzung einer verschärften Klimapolitik die lokale Luftverschmutzung nicht bereits optimal reguliert war. 
Instrumente in der Zukunft gezielt $\mathrm{zu}$ verbessern und vorhandene Potentiale $\mathrm{zu}$ realisieren. Das Argument schließt somit auf unzulässige Weise von einer spezifischen, zweitbesten Vergangenheit auf die Unmöglichkeit einer erstbesten Zukunft, bzw. auf die Unmöglichkeit, diese im Zweitbesten mithilfe konventioneller Klimapolitik zu erreichen. Die Unvereinbarkeit von Klimaschutz und Wachstum folgt jedoch auch dann nicht, wenn man die vorgeschlagene sorgfältigere Unterscheidung zwischen Erst- und Zweitbestem trifft: Sowohl erstbeste Potentiale als auch zweitbeste Instrumente wären vorhanden deren politische Umsetzung ist das zentrale Problem.

Der aktuelle Forschungsstand zu zukünftig möglichen erstbesten Emissionsreduktionen („optimalen Dekarbonisierungspfaden“) für die Weltwirtschaft ist, dass bei großzügiger Verfügbarkeit aller relevanten Technologien zumindest die Erreichung eines $2^{\circ} \mathrm{C}$-Ziels mit weiterem Wirtschaftswachstum vereinbar ist. Dies geht aus den Ergebnissen des letzten Sachstandsbericht des Intergovernmental Panel on Climate Change (IPCC) hervor, welches den Forschungstand zum Klimawandel regelmäßig zusammenfasst (IPCC, 2014). Diese Ergebnisse beruhen zu großen Teilen auf Simulationen von „CostEffectiveness Integrated-Assessment Modellen“ (Clarke et al. 2014). ${ }^{7}$ Zwar kostet auf optimalen Dekarbonisierungspfaden Klimaschutz größenordnungsmäßig $10 \%$ des globalen Outputs über das gesamte 21. Jahrhundert (ohne Berücksichtigung der vermiedenen Schäden). Jedoch rechnen die Modelle dies unter der Annahme einer Verdopplung bis Verviereinhalbfachung der Weltwirtschaft im Laufe dieses Jahrhunderts (Clarke et al. 2014). Klimaschutz im Erstbesten erfordert also definitiv keine Schrumpfung der Wirschaft. Das $2^{\circ} \mathrm{C}$-Ziel mit weiterem Wirtschaftswachstum zu vereinbaren erfordert jedoch auch die weitgehende Nutzung gesellschaftlich umstrittener Technologien wie der Abscheidung und unterirdischer Speicherung von $\mathrm{CO}_{2}$ (CCS) und der Erzeugung ,negativer Emissionen“ durch die Kombination von CCS mit der Energieerzeugung aus Biomasse. ${ }^{8}$ Dabei werden auch die Auswirkungen für die globale Landnutzung und den damit zusammenhängenden Umweltproblemen (siehe oben) weitgehend berücksichtigt (Weyant 2017). Man kann mit guten Gründen der Ansicht sein, dass solche Technologien entweder zu spekulativ oder zu risikoreich sind, und insbesondere eine Gefahr für die Ernährungssicherheit darstellen. Allerdings ergeben sich selbst bei einer Vermeidung von CCS zwar höhere Vermeidungskosten, diese liegen

7 Zur Kritik an den technischen Annahmen dieser Modelle siehe Abschnitt „Diskussion: Einordnung der Argumente“. Wären die Annahmen in den CEA-IAMs unstimmig, so gäbe es Gründe, an der Vereinbarkeit von Wirtschaftswachstum und Klimawandel zu zweifeln.

${ }^{8}$ Für das zusätzlich im Pariser Klimaschutzabkommen erwähnte $1,5^{\circ} \mathrm{C}$-Stabilisierungsziel legen neueste Forschungsarbeiten (IPCC 2018) nahe, dass selbst dies bei weiterem Wirtschaftswachstum im Erstbesten noch erreichbar wäre, allerdings nur mit erheblichem Einsatz von Technologien, die der Atmosphäre $\mathrm{CO}_{2}$ wieder entziehen können. In der Diskussion um Umweltziele, die mit einer etwaigen Schrumpfung der Wirtschaftsleistung einhergehen würden, müssten jedoch die vermiedenen Umweltschäden gegen möglichen negativen Folgen einer raschen Änderung der Wirtschaftsweise abgewogen werden (Dietz et al. 2018). 
jedoch immer noch eine Größenordnung niedriger als die Kosten einer pauschalen Schrumpfung der Weltwirtschaft.

Diesen globalen Kostenvergleich illustrieren wir nun an einer einfachen Beispielrechnung für Deutschland, die selbstverständlich eine Modellierung des Energiesektors nicht ersetzen kann: 2012 wurden in Deutschland pro emittierter Tonne Treibhausgase rund $2.890 €$ erwirtschaftet ${ }^{9}$ (eigene Berechnung nach Europäische Kommission, 2017). Zum Vergleich kann bei einer nahezu kompletten Umstellung des deutschen Stromsystems auf erneuerbare Energien bis 2050 (unter Berücksichtigung von Nutzungs- und Naturschutzrestriktionen) mit einer Emissionsminderung im Stromsektor von 95\% gegenüber 1990 bei Vermeidungskosten zwischen -15€ und 125€ pro Tonne $\mathrm{CO}_{2}$ gerechnet werden (je nach Preisentwicklung fossiler Brennstoffe; Agora Energiewende 2017). ${ }^{10}$ Umgekehrt scheint eine Minderung des globalen Wirtschaftswachstums die Erreichung der Dekarbonisierungsziele nicht in nennenswertem Maße zu erleichtern: Wird für Dekarbonisierungspfade ein deutlich geringeres Wirtschaftswachstum modelliert, so ändert sich nur wenig in Bezug auf die Dekarbonisierungsleistung, welche der Technologiemix aus regenerativen Energien, Effizienzsteigerungen und ggf. negativen Emissionen leisten muss (Jakob und Edenhofer, 2014). Darum scheint eine Absenkung des Wirtschaftswachstums nicht prioritär, unabhängig davon, wie man die Abwägungen in der globalen Landnutzung zwischen Klimaschutz und Ernährungssicherheit sieht.

Der optimale Klimaschutzbeitrag verschiedener Wirtschaftsbereiche ist nicht allein mit technologischen Lösungen zu erreichen. Optimale Dekarbonisierungspfade beinhalten nicht nur Verbesserungen der Energieeffizienz, sondern auch Verhaltensänderungen auf der Nachfrageseite. So wird in vielen Sektoren die Energienachfrage deutlich sinken müssen, insbesondere für Mobilität, Ernährung und im Gebäudesektor (Clarke et al., 2014). Dies kann nur mit einer Reduktion der Nachfrage von besonders schwer zu dekarbonisierenden Produkten erreicht werden, für die es wahrscheinlich bis zur Mitte des Jahrhunderts keine adäquaten Substitute gibt. In der Folge könnten kleinere Wirtschaftssektoren durchaus schrumpfen (sowohl in physischen als auch monetären Einheiten), beispielsweise die Fleischproduktion oder der Flugverkehr.

Abbildung 1 veranschaulicht das gegenwärtige Verhältnis von Wirtschaftsleistung und Emissionen verschiedener Sektoren in Deutschland: Der Energiesektor ist bei etwa 3\% Anteil an der Wertschöpfung für rund 50\% der $\mathrm{CO}_{2}$-Emisisonen verantwortlich. Daneben gibt es eine Reihe von weiteren Sektoren, deren Emissionsanteile im einstelligen

${ }^{9}$ Zur Frage, ob dadurch ein gesellschaftlicher Wohlfahrtsgewinn entsteht, siehe Textabschnitt „Wollen wir Wachstum aufgeben?"

${ }^{10}$ Der Stromerzeugung kommt in Deutschland mit 35\% Emissionsanteil in 2017 (UBA 2019) eine Schlüsselrolle zu; je nach Dekarbonisierungsstrategie ist sie auch für Emissionsreduktionen in anderen Sektoren, etwa die Elektrifizierung des Verkehrs, von zentraler Bedeutung. Die $\mathrm{CO}_{\mathrm{n}^{-}}$ Vermeidungskosten in anderen Sektoren sind von vergleichbarer Größenordnung (vgl. IPCC Technical Summary 2014). 
Prozentbereich die jeweilige Wirtschaftsleistung deutlich überwiegen, z.B. chemische Industrie, Luftfahrt, und Zementproduktion. Eine effiziente Regulierung sollte möglichst gezielt bei diesen Sektoren ansetzen. Für alle anderen Sektoren liegt der Anteil am $\mathrm{CO}_{2^{-}}$ Ausstoß unter $2 \%$ mit teilweise sehr hohen Anteilen an der Wirtschaftsleistung, insbesondere bei Forschung und Entwicklung, Immobilien und Gesundheit. 


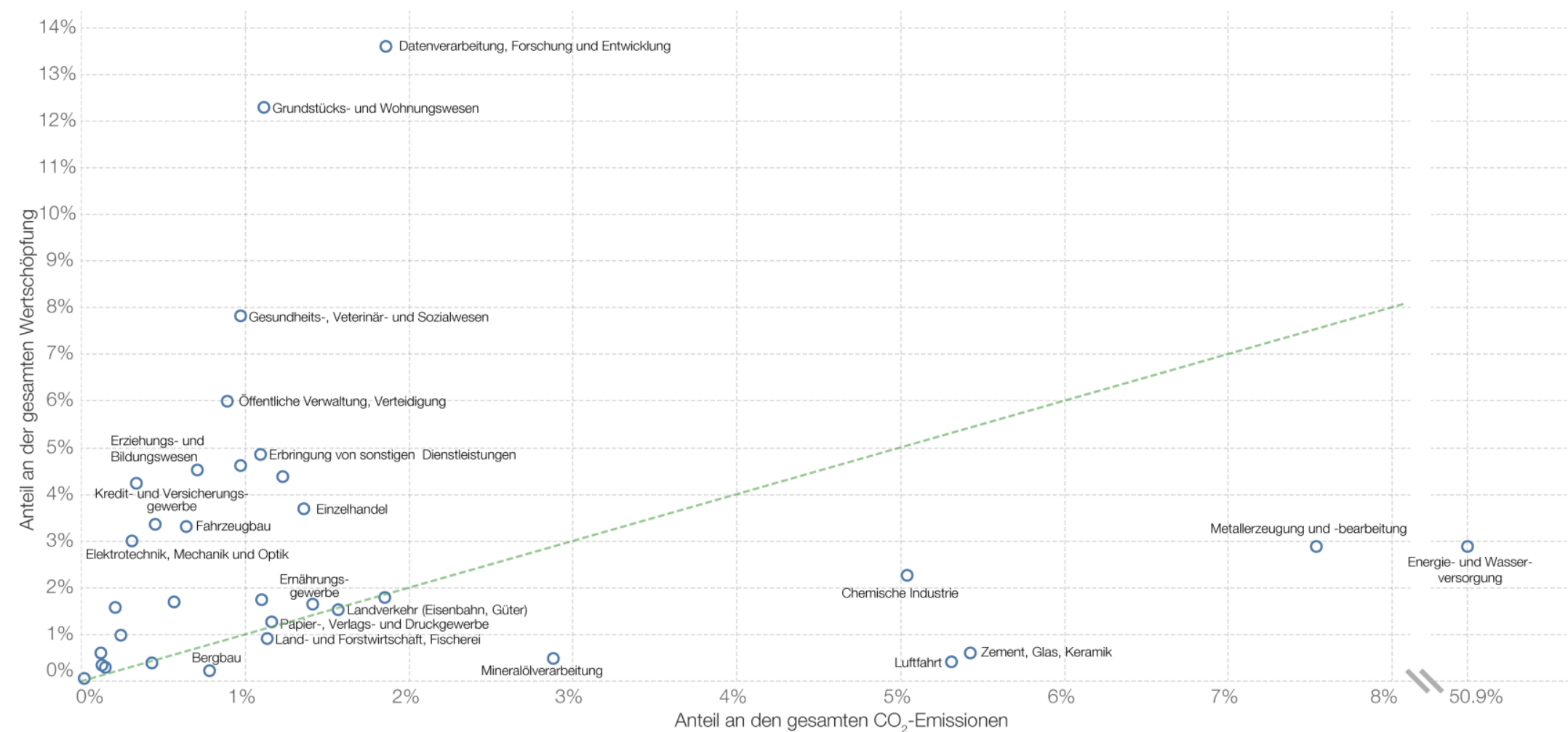

Abbildung 1: Anteil von Sektoren an der gesamten Wertschöpfung und an den $\mathrm{CO}_{2}$-Emissionen für Deutschland im Vergleich (mit gestauchter horizontaler Achse). Datenquelle: World Input-Output Database (2013). Daten für 2009, Darstellung ohne Direktemissionen der Haushalte. 
$\mathrm{Zu}$ den Möglichkeiten des zweitbesten Klimaschutzes, die offensichtlich vorhandenen technologischen Potentiale auch tatsächlich mit realistischen Politikinstrumenten auszuschöpfen, lässt sich feststellen, dass die bisherigen Instrumente zwar vollkommen unzureichend waren, die notwendigen Verbesserungen aber bekannt sind: Es fehlt nicht an Instrumenten für effiziente, gezielte Emissionsreduktionen, sondern an politischem Willen, diese umzusetzen.

Dafür seien fünf Beispiele genannt. Erstens könnten in vielen Ländern weiterhin Subventionen abgeschafft werden, die fossile Energieträger oder klimaschädliche Produktions- und Konsummuster fördern („Pendlerpauschale“). Zweitens haben gegenwärtige $\mathrm{CO}_{2}$-Preissysteme eine unzureichende geographische und sektorale Abdeckung und sind viel zu wenig ambitioniert, um die für ein $2{ }^{\circ} \mathrm{C}$-Ziel nötigen Emissionsminderungen zu erreichen (Stiglitz et al., 2017). Darüber hinaus sind regulatorische Lösungsansätze und politische Grundsatzentscheidungen wie der Kohleausstieg oder Effizienzrichtlinien im Transportsektor wirksame Mittel, wenn marktbasierte Instrumente politisch nicht durchsetzbar sind. Drittens müssten langlebige Investitionen in den Schlüsselsektoren Energie, Transport und Gebäude klimafreundlicher werden, um einen „Lock-In“ weiterer zukünftiger Emissionen zu verhindern (Guivarch und Hallegatte, 2011), gerade bei öffentlichen Infrastrukturinvestitionen. Viertens müssten vielfältige Marktstrukturen und Regularien angepasst werden, die gegenwärtig auf emissionsintensive Technologien zugeschnitten sind, z.B. in Energienetzen und -märkten, Verkehrs-, Raumplanungs- und Bauverordnungen. Fünftens könnte eine bessere Berücksichtigung des tatsächlichen, nicht immer rationalen Verhaltens der Marktteilnehmer die Effektivität gezielter Klimapolitik deutlich und kostengünstig steigern (Gillingham und Palmer, 2014).

Wir erläutern die Unterscheidung zwischen technischer Machbarkeit und wirschaftspolitischer Regulierung nun noch knapp am Beispiel des notorisch schwer zu dekarboniserenden Verkehrssektors: Eine optimale Transformation dieses Sektors erfordert eine Reduktion der Gesamtzahl aller Fahrten und Wechsel der Verkehrsträger durch Verhaltensänderungen, eine Steigerung des Anteils an effizienteren Fahrzeugen und Motoren, Elektrifizierung sowie den Einsatz weiterer klimafreundlicher Treibstofffe, Umbau der Infrastruktur und Änderungen im Städtebau (Sims et al. 2014). Diese technischen Änderungen müssen mit verkehrspolitischen Instrumenten durchgesetzt werden, die von Steuern auf Treibstoff und City-Mautsystemen sowie Emissionsstandards über andere Bebauungs- und Verkehrswegepläne bis hin zu Parkraumbewirtschaftung und Informationskampagnen reichen (dazu ausführlich: Banister, 2008; Creutzig et al., 2015; Mattauch et al., 2016; Sims et al. 2014).

Dass die nötigen Verbesserungen bestehender Politikinstrumente oder die Durchsetzung neuer Formen der Regulierung der Wirtschaft noch nicht stattgefunden haben, kann auf eine Vielzahl von Gründen zurückgeführt werden: So spielen kurzfristiges Denken, Unwissenheit und Unsicherheit bei KonsumentInnen, unzureichende Informationen und Fähigkeiten staatlicher AkteurInnen, Strukturkonservatismus in Wirtschaft und Politik, techno-soziologischer Lock-In, Verteilungskonflikte, Lobbyarbeit oder Korruption und verschiedene Wertesysteme eine Rolle. Auf internationaler Ebene erschweren weiterhin zwischenstaatliches Trittbrettfahrer-Verhalten, sowie Gerechtigkeits- und 
Verfügungskonflikte die Kooperation im Klimaschutz (Edenhofer, Flachsland und Brunner, 2011). Zweifellos ist die Überwindung dieser Barrieren extrem herausfordernd. Andererseits wäre auch eine Politik der gesamtwirtschaftlichen Stagnation oder Schrumpfung mit immensen politischen und institutionellen Hürden verbunden, die von den WachstumskritikerInnen zumeist nicht im Detail betrachtet werden. Dies lässt sich gut anhand von Verteilungsfragen illustrieren.

Die politische Machbarkeit gezielter Klimapolitik hängt stark von Verteilungseffekten $\mathrm{ab}$, insbesondere bei stark wahrgenommenen und auf bestimmte Gruppen konzentrierten Einbußen, und wenn diese Gruppen gut organisiert und politisch einflussreich sind. Beispielsweise können Kürzungen klimaschädlicher Subventionen und ein höherer, umfassender $\mathrm{CO}_{2}$-Preis über Strom-, Heizungs- und Benzinkosten niedrige Einkommen relativ stärker belasten als hohe Einkommen. Dem kann mit einer entsprechenden Rückverteilung der eingesparten Mittel bzw. zusätzlichen Einnahmen entgegengewirkt werden, die allerdings auch eine hohe Sichtbarkeit aufweisen muss um politisch akzeptabel zu sein (Klenert et al., 2018). Außerdem reduziert ein hoher $\mathrm{CO}_{2}$-Preis die Renten von Besitzern fossiler Ressourcen und damit betriebener Anlagen (Siegmeier et al. 2018), so dass auch hier aus Gründen der politischen Machbarkeit Kompensationen nötig werden können. Zusätzliches Wirtschaftswachstum würde eher helfen, dafür die nötige Finanzierug zu sichern. Eine gesamtwirtschaftliche Schrumpfung würde zwar die meisten Bevölkerungsgruppen in ähnlicher Weise betreffen, was Verteilungsdebatten möglicherweise entschärfen könnte. Allerdings ist selbst ohne Verteilungseffekte nicht zu erwarten, dass die Bevölkerungsmehrheit ohne Weiteres einen Teil ihres Wohlstands aufgeben würde.

Die Unvereinbarkeit von Klimaschutz und Wirtschaftswachstum gilt weder für den Fall prinzipiell unzureichender Technologien noch für den Fall unüberwindlicher politischinstitutioneller Hindernisse konventioneller Klimapolitik. ${ }^{11}$ Auch sehr ambitionierte Umweltschutzziele können effizienter durch eine gezielte Regulierung der verantwortlichen Sektoren erreicht werden als durch Maßnahmen, die bei der gesamten Wirtschaftsleistung ansetzen. Die Forderung nach einer Schrumpfung der Wirtschaftsleistung verstellt den Blick auf die zentrale Frage, die aus argumentationslogischer Sicht, jedoch insbesondere im Sinne schnellen, effektiven Klimaschutzes noch vorher zu klären wäre - nämlich danach, ob und wie Umweltpolitik in Zukunft unter Berïcksichtigung politischer und institutioneller Hürden ambitioniert und effizient ausgestaltet werden kann.

${ }^{11}$ Umgekehrt: Wäre die nötige Entkopplung des Wachstums von Emissionen physisch-technisch unmöglich, was sie nach IPCC (2014) aber nicht ist, so wäre es selbstverständlich auch unter wie auch immer gewählten Politikinstrumenten richtig, dass Klimaschutz nicht mit Wirtschaftswachstum vereinbar ist. 


\section{Wollen wir Wachstum aufgeben? Eine empirische und normative Einordnung}

Mit psychologischen Studien zum subjektiven Wohlergehen der Bevölkerung - also darüber, wie glücklich und bedeutungsvoll Menschen ihr Leben wahrnehmen - wird in der Wachstumskritik begründet, dass eine wachstumsorientierte Wirtschaftspolitik das Glück der Gesellschaft nicht steigert. Damit wird unabhängig von vermuteten natürlichen Grenzen des Wirtschaftswachstums die Frage aufgeworfen, ob weiteres Wirtschaftswachstum in reichen Ländern überhaupt erstrebenswert sei. Dies geschieht auch im Zusammenhang mit Materialismuskritik: Bestimmte Ausprägungen der Konsumgesellschaft wie etwa Stress in der Arbeitswelt, Depressionen und mangelnder sozialer Zusammenhalt werden oftmals auf einen immer größeren Stellenwert des Konsums in der Gesellschaft zurückgeführt.

Das Argument, dass Wirtschaftswachstum nicht erstrebenswert sei, da es Glück und Bedeutung nicht steigere, lässt sich wie folgt strukturieren:

1. Prämisse: Wirtschaftswachstum steigert Glück und Bedeutung nicht.

2. Prämisse: Glück oder Bedeutung zu steigern ist das oberste Ziel von

Wirtschaftspolitik.

Schlussfolgerung: Die Wirtschaft soll nicht weiter wachsen.

Das Argument ist nicht ohne weitere Annahmen gültig. Zunächst folgt die Konklusion aus den beiden Prämissen nur dann, wenn sich Glück oder Bedeutung durch gezieltes Nicht-Wachstum besser steigern ließe. Darüber hinaus wird das Argument nur überzeugend sein, wenn seine Prämissen plausibel sind. Diese sind unterschiedlicher Natur: Prämisse 1 trifft eine empirische Aussage darüber, dass herkömmliches Wachstum Glück und Bedeutung nicht steigert. Prämisse 2 drückt aus, dass der normative Standpunkt, dass Glück- oder Bedeutungszuwachs das Hauptziel der Wirtschaftspolitik bilden, überzeugend ist.

Wir zeigen im Folgenden, dass die erste Prämisse für bestimmte Glücksmaße zutrifft, wenn auch nicht für alle und ordnen anschließend die zweite Prämisse in die Wohlfahrtstheorie ein. Allerdings gelten die empirischen Aussagen naturgemäß nur für das historisch erfolgte Wachstum. Das obige Argument ist, unter Annahme beider Prämissen, also nur für bisheriges Wachstum überzeugend. Und selbst wenn vergangenes Wirtschaftswachstum bisher das Glück der Bevölkerung nicht gesteigert hat und Glückssteigerung Ziel von Wirtschaftspolitik sein soll, so impliziert das noch nicht, dass zukünftiges Wirtschaftswachstum nicht effektiver zur Glückssteigerung beitragen könnte als vergangenes.

\section{Empirische Analyse}

Um die tradierte Hypothese der Wachstumskritik, „Wirtschaftswachstum macht die Menschen nicht glücklicher" empirisch zu überprüfen, bedarf es zunächst einer begrifflichen Schärfung: In der Glücksforschung unterscheidet man zwei Formen des 
subjektiven Wohlbefindens („Glück“). Affektives Glück beschreibt die emotionale Güte einer individuell erlebten Erfahrung (Kahneman und Krueger, 2006) und wird zum Beispiel durch die „Day Reconstruction Method“ erfasst, die durch eine möglichst kurze Zeitspanne zwischen Erlebnis und Befragung die intersubjektive Vergleichbarkeit steigert (Kahneman et al., 2004). Lebenszufriedenheit bezeichnet im Gegensatz dazu eine reflektierte Einschätzung der gesamten Lebenssituation eines befragten Individuums. Individuen sollen beispielsweise ihr Leben entlang einer festgelegten Skala bewerten, an deren Enden das für sie bestmöglichste und schlechtmöglichste Leben stehen („Ladder“Methode, vgl. Kahneman und Deaton, 2010). Die Ergebnisse der Glücksforschung sind insofern robust und zuverlässig, als dass sie mit plausiblen Charakteristika für Glück korrelieren, wie einer Beschreibung als ,glücklich“ durch Freunde und Bekannte, der Häufigkeit eines echten Lächelns, sowie niedrigen Suizidraten als auch bestimmten Hirnaktivitäten (Kahneman und Krueger, 2006; Layard, 2011).

Um die Bedeutung des Wirtschaftswachstums für das subjektive Wohlbefinden der Menschen zu ergründen, betrachten wir die Effekte einer Einkommenserhöhung für Individuen und anschließend die Entwicklung der Durchschnittseinkommen für Gesellschaften.

Kahneman und Deaton (2010) stellen einen signifikanten Effekt einer Einkommenserhöhung auf das affektive Glück von Individuen nur bis zu einem Einkommen von rund 75.000 US\$ fest. Das tägliche Wohlbefinden der Menschen verbessert sich also ab einem gewissen Einkommen nicht mehr. In der Gesamtbeurteilung der Lebenssituation tritt keine derartige Grenze auf: Bei der Anwendung der LadderMethode wird über alle Einkommensklassen hinweg eine positiver Zusammenhang von Einkommenserhöhungen und Lebenszufriedenheit festgestellt (Kahneman und Deaton, 2010).

Im Ländervergleich wird zudem ein positiver Zusammenhang zwischen Durchschnittseinkommen und Lebenszufriedenheit festgestellt (Easterlin et al., 2010). Betrachtet man jedoch die langfristige Entwicklung der Durchschnittseinkommen innerhalb eines Landes, erscheinen die Effekte von Einkommenssteigerungen auf die Lebenszufriedenheit verschwindend gering (Easterlin et al., 2010). Dieser Gegensatz ist der Gehalt des sogenannten „Easterlin-Paradoxon“. Unter der Prämisse, dass Glück im Sinne von Lebenszufriedenheit das Ziel von Wirtschaftspolitik ist, lässt diese Erkenntnis konventionelles Wirtschaftswachstum nicht erstrebenswert erscheinen.

In Fachkreisen wird die Gültigkeit des Easterlin-Paradoxons bisweilen angezweifelt. KritikerInnen bemängeln unter anderem den geringen Stichprobenumfang in Easterlins Studien und finden stattdessen eine schwache (aber statistisch signifikante) positive Korrelation zwischen absoluten Einkommenssteigerungen und Glück (Sacks, Stevenson und Wolfers, 2012). Aus einer möglicherweise positiven Korrelation zwischen absoluten Einkommenssteigerungen und Glück folgt jedoch nicht, dass konventionelles Wirtschaftswachstum das primäre politische Mittel zur Steigerung des Glücks sein sollte. Wenn überhaupt, so können absolute Einkommenssteigerungen nur einen Teil der Varianz der individuellen Glücksniveaus erklären.

Zur Erklärung der scheinbar widersprüchlichen Ergebnisse zum Zusammenhang von Einkommen und Glück lassen sich zunächst Statuskonsum und Gewöhnungseffekte 
heranziehen (Layard, 2011): Menschen messen ihre Zufriedenheit im sozialen Vergleich mit anderen und tendieren dazu ihre materiellen Wünsche nach oben zu korrigieren, sobald sie sich an ihr eigenes Wohlstandsniveau gewöhnt haben. Wird zum einen das Umfeld reicher, so hat dies negative Effekte auf das eigene Glücksniveau, weil Menschen sich miteinander vergleichen. Zudem unterschätzen Menschen, wie stark sie sich an Neuanschaffungen gewöhnen (Layard 2011). Falls nur relative Einkommensunterschiede die Zufriedenheit steigerten, nicht aber absolute, so ließe sich das Easterlin-Paradoxon auflösen.

Weiterhin ist zwar davon auszugehen, dass Menschen im Normalfall anstreben, ihr „Glück“ zu mehren, zumindest im Sinn der Lebenszufriedenheit. Dennoch ist in Kognitionswissenschaft und Glücksforschung breit anerkannt, dass es eine Vielzahl von Gründen gibt, warum Menschen ein höheres Einkommen nicht zur Steigerung ihres eigenen Glücks einsetzen wollen oder können. Auf der individuellen Entscheidungsebene zeigt sich, dass Menschen nicht immer die Option wählen, die sie tatsächlich glücklicher macht (Hsee und Hastie, 2006). Zum einen liegt das an falschen Vorhersagen darüber was glücklich macht, etwa aufgrund von Selbstüberschätzung oder falscher Erinnerung, oder weil Menschen nicht nach ihren Vorhersagen, was glücklich macht, handeln, etwa wegen Willensschwäche (Hsee und Hastie, 2006). Außerdem gibt es Situationen, in denen Menschen andere Ziele verfolgen können als glücklich zu sein, wie etwa sich mehr für ihre Familie oder für ihre eigene Zukunft einzusetzen (Benjamin et al., 2012). Dies weist darauf hin, dass Bedeutung eine von Glück recht unabhängige Qualität menschlichen Lebens ist (Baumeister et al., 2013).

Aus der Aussage, dass Wirtschaftswachstum nicht zu einer Steigerung der Lebenszufriedenheit beitrage, leiten viele WachstumskritikerInnen ab, dass Wirtschaftswachstum nicht erstrebenswert sei. Dies erfordert jedoch, dass auch die Negation eben jener Aussage gilt: „Kein Wachstum macht die Menschen nicht unglücklicher“. Selbst wenn man nur Studien betrachtet, die die Korrelation zwischen Lebenszufriedenheit und Wirtschaftswachstum anzweifeln, lässt sich nicht ableiten, dass eine Schrumpfung der Wirtschaftsleistung und deren Folgen nicht mit Rückgängen der Zufriedenheit verbunden wären. Ähnliches gilt für weitere sozioökonomische, gesamtgesellschaftlich relevante Faktoren die im Nationenvergleich mit Glück korrelieren, wie zum Beispiel der Grad an Gleichheit, sozialstaatlicher Versorgung oder sozialem Vertrauen in einer Gesellschaft (Layard, 2011): Diese Faktoren lassen sich sicherlich durch eine fokussierte Wirtschafts- und Sozialpolitik besser fördern als durch die allgemeine Steigerung des Wirtschaftswachstums. Ob sie jedoch trotz eines Verzichts auf Wachstum in gleichem Maße gefördert werden können, kann man bezweifeln.

Umgekehrt kann für einige politische Maßnahmen zur Steigerung des Glücks in der Gesellschaft nicht von vornherein ausgeschlossen werden, dass sie auch zu Wirtschaftswachstum führen. Beispielsweise würde eine starke Senkung der Verbreitung von Depression in der Bevölkerung sicherlich zu höherer Arbeitsproduktivität führen, die sich in Wirtschaftswachstum niederschlagen könnte. ${ }^{12}$

12 Wenn allerdings das Glück gegenwärtig vor allem deshalb nicht maximal ist, weil die Bevölkerung zu viel arbeitet, kann es in der Transition zu einer Gesellschaft mit weniger 
Zusammenfassend deuten die empirischen Erkenntnisse der Glücksforschung darauf hin, dass zumindest ab einer bestimmten Grenze Einkommenserhöhungen das affektive Glück der Menschen nicht weiter erhöhen. Darüber hinaus sind Korrelationen zwischen Wirtschaftswachstum und dem alternativen Glücksmaß der Lebenszufriedenheit im langfristigen Ländervergleich umstritten. Wirtschaftswachstum wurde somit lange in seiner wirtschaftspolitischen Wirkung auf das Glück der Bevölkerung überschätzt. Jedoch ergibt sich daraus nicht automatisch ein überzeugendes Argument zu Gunsten der Schrumpfung oder Stagnation.

\section{Normative Analyse}

Ebenso entscheidend für eine Bewertung, ob weiteres Wirtschaftswachstum wünschenswert sei, ist die normative Grundlage für Wirtschaftspolitik: Die zweite Prämisse des obigen Arguments gibt an, dass die Steigerung der Lebenszufriedenheit, des affektiven Glücks oder der Bedeutung das Ziel wirtschaftspolitischen Handelns sein soll. Die neoklassische Volkswirtschaftslehre stellt jedoch keines dieser Konzepte ins Zentrum ihrer Theorie dessen, was das Ziel von Wirtschaftspolitik sei. Sie nimmt stattdessen an, dass die Erfüllung von Präferenzen der richtige Maßstab für die Bewertung von Wirtschaftspolitik ist - ein von der Steigerung der Lebenszufriedenheit abzugrenzender Standpunkt (Crisp, 2001; Fleurbaey und Blanchet, 2013; Loewenstein und Ubel, 2008). In seiner Reinform ist der normative Standpunkt der neoklassischen Theorie: „Was auch immer Menschen wählen, stellt sie besser." (Loewenstein und Ubel, 2008, S.1795). Präferenzen werden nach dieser Auffassung also durch tatsächliche Entscheidungen „enthüllt“. Es gilt beispielsweise nicht alternativ als ihre Präferenz, was Menschen verbalisieren.

Aus moralphilosophischer Perspektive ist dieser Standpunkt nicht selbst-evident. Es bedarf einer moralphilosophischen Erörterung, warum Wirtschaftspolitik gerade nach diesem und nicht nach einem anderen normativen Standpunkt bewertet werden soll (Hausman, 2012). Tatsächlich wird in der zeitgenössischen Wohlfahrtstheorie breit diskutiert, ob nun die Erfüllung von Präferenzen, die Steigerung substantieller Ziele wie Glück oder beispielsweise ein Fähigkeitsansatz nach Sen und Nussbaum (Nussbaum, 2001; Sen, 1985) die Grundlage wirtschaftspolitischen Handelns sein soll (Fleurbaey und Blanchet, 2013). Bezüglich der spezifischen Position, das Glück der Bevölkerung steigern zu wollen, hier nur knapp die wichtigsten Punkte (siehe ausführlich: Fleurbaey und Blanchet, 2013; Loewenstein und Ubel, 2008; Mattauch und Hepburn, 2016; Sen, 1985): Einerseits sehen Vertreter der Präferenzkonzeption die Idee von Glück als Politikziel als "paternalistisch" an. ${ }^{13}$ Menschen können legitimerweise andere Ziele

Arbeitszeit durchaus zu einer Schrumpfung des wirtschaftlichen Outputs kommen (Bilancini und D'Alessandro, 2012).

13 Eine Interpretation einer freiheitlichen Grundordnung ist die Beschränkung staatlicher Intervention auf lediglich jene Aktivitäten, die Dritte schädigen. Nimmt man die Förderung des gesellschaftlichen „Glücks“ als primäres Politikziel an, so scheint es folgerichtig, dass der Staat auch in Wahlentscheidungen eingreifen könnte, die nur das Individuum selbst betreffen (z.B. Ernährung, Bewegung, Konsumverhalten). Diese Frage scheint aber unabhängig davon, welche 
verfolgen, als nach Glück zu streben. Darüber hinaus ist Glück auf Grund psychologischer Gewöhnungseffekte kein guter Indikator für materielle Entbehrungen (Sen, 1985): Armutsbekämpfung mag sozial selbst dann erwünscht sein, wenn Armut gar nicht unglücklich macht. Andererseits sehen Vertreter der Glücksmaximierung sehr große praktische Schwierigkeiten darin, verlässliche Aussagen über individuelle Präferenzen zu treffen, da Menschen bei ihren Entscheidungen vielfach kognitiven Verzerrungen unterliegen (Loewenstein und Ubel 2008). Zudem haben diejenigen Fälle, in denen Menschen ihr Glück nicht anstreben, wenig wirtschaftspolitische Relevanz (Layard 2011, Greene 2013).

Für die Einordnung der Wachstumskritik sei insbesondere angemerkt: Die meisten Menschen dürften die extreme Form des Präferenzansatzes - ,was auch immer Menschen wählen, stellt sie besser“ - nicht als plausibel empfinden, insbesondere angesichts moderner „Zivilisationskrankheiten“ wie Übergewicht. Die Neoklassik begründet eine Entscheidung lediglich damit, dass das Individuum eine Präferenz für die ausgewählte Option hatte (Lancaster, 1966) und sieht Präferenzen als vollständig und stabil an (Ariely et al. 2006). Präferenzen sind hingegen, im Sprachgebrauch jenseits der formalen Ökonomik, immer Gegenstand rationaler Überlegung und Begründung durch das Individuum und nicht unveränderlich gegeben. Darüber hinaus ist es unklar, wie der Präferenzansatz angewendet werden kann, wenn politische Maßnahmen Präferenzen verändern. In diesem Fall kann ein naiver Präferenzansatz die Veränderung nicht mehr bewerten. Welche Präferenzen befördert werden sollen ist jedoch eine Frage, über die Gesellschaften sich verständigen können, beispielsweise in der Bildungspolitik (Gintis, 1974), aber auch in der Umweltpolitik (Mattauch und Hepburn, 2016).

Wenn wachstumskritische Stimmen also oftmals von der Position der Glückssteigerung ausgehend argumentieren, dass weiteres Wirtschaftswachstum nicht erstrebenswert sei, so sind sie sich der Probleme des Präferenzansatzes der Ökonomik sicherlich mindestens implizit gewahr. Entscheidend für die Debatte darüber, ob weiteres Wirtschaftswachstum erstrebenswert sei, erscheint somit: Diese sollte über gesellschaftliche Ziele geführt werden, anstatt lediglich zu bestätigen, dass das Bruttoinlandsprodukt keine sinnvolle Zielmarke darstellt. Verhaltensökonomik und Glücksforschung liefern wichtige Beiträge um eine Kritik an materialistischem Denken und der Konsumgesellschaft zu fundieren. Auch wenn die Position eingenommen wird, dass Wirtschaftspolitik das Glück steigern solle, so ist zu vermuten, dass Wachstum zur Steigerung des Glücks effizienter als bisher eingesetzt werden und somit grundsätzlich auch für die Glückssteigerung hilfreich sein kann.

Politikmaßnahmen grundsätzlich zweckmäßig sind, etwa auf Marktpreise erhobene Steuern oder direkte Verbote. 


\section{Diskussion: Einordnung der Argumente}

Der vorherige Abschnitt hat zwei überzeichnete und in ihrer Reinform in der Literatur kaum vertretene, aber im öffentlichen Diskurs verbreitete Argumente für die Unmöglichkeit oder Unerwünschtheit weiteren Wachstums betrachtet, um an ihnen Fehlschlüsse im Postwachstumsdiskurs aufzuzeigen. Die dezidierte Forderung nach einer Wachstumsrücknahme zum Schutz der Umwelt oder zur Steigerung der Lebensqualität ist nicht leicht zu begründen. Das heißt aber nicht, dass Varianten dieser Argumente nicht schlüssig und für die konventionelle Volkswirtschaftslehre bedenkenswert sein könnten. Wir stellen drei verwandte kritische Sichtweisen auf das Wirtschaftswachstum vor und bewerten sie knapp.

Erstens bezweifelt eine weitere Sichtweise zwar nicht grundsätzlich, dass Wirtschaftswachstum mit der Einhaltung „planetarer Grenzen“ vereinbar sein könnte. Trotzdem sei über eine organisierte Schrumpfung der Wirtschaft für den Fall nachzudenken, dass die bisherigen Prognosen über die technischen Möglichkeiten sich nicht bewahrheiteten, insbesondere auch bezüglich spekulativer Risikotechnologien wie etwa der Erzeugung „negativer Emissionen“ durch Biomassenutzung und $\mathrm{CO}_{2}$ Abscheidung (Fuss et al., 2014; Creutzig, 2016). So lässt sich kritisch hinterfragen, inwiefern die in der Politikberatung genutzten Integrated Assessment Modelle objektive und verlässliche Vorhersagen treffen können (Ellenbeck, 2017; Pissarskoi 2016; Pindyck, 2013; Weyant, 2017). Es könnte sein, dass diese fehlerhafte Annahmen über die Technologie-, Bevölkerungs- und Nachfrageentwicklung beinhalten oder bestehende Interdependenzen zwischen Wirtschaftssektoren, die Wirtschaftswachstum und Dekarbonisierung doch unvereinbar machen, bisher nicht modellieren. Diese Sichtweise ist schlüssig — allerdings ist fraglich, ob die Problematik angesichts der großen Hürden bei der Durchsetzung von Klimaschutzmaßnahmen im Zweitbesten und des bereits bestehenden enormen Detailreichtums der Modelle einen prioritären Forschungsbedarf darstellt.

Eine verwandte, offenbar durch die „Wachstumsfixierung“ einiger politischer AkteurInnen ausgelöste Sichtweise ist, dass die Gesellschaft unabhängiger vom Wirtschaftswachstum werden müsse (Seidl und Zahrnt, 2010, S.24ff). Eine Gesellschaft müsse also beispielsweise den Kohleausstieg aus Gründen des Umweltschutzes auch dann durchsetzen können, wenn es damit zu Wachstumseinbußen kommt. Diese Position ist allerdings in der Wissenschaft nicht sonderlich kontrovers. Sie ist vielmehr eine Grundannahme der Umweltökonomik (vgl. Perman et al. 2003), obschon sie politisch durchaus nicht selbstverständlich ist (vgl. Raworth, 2017). Es lässt sich hingegen bezweifeln, ob wirklich die Sorge um das allgemeine Wirtschaftswachstum effektive Umweltpolitik erschwert, oder vielmehr die damit verbundenen Verteilungskonflikte.

Zweitens fragt manche Wachstumskritik, was mit gesellschaftlichen Problemfeldern wie den Auswirkungen des demographischen Wandels auf das Kranken- und 
Rentenversicherungssystem geschehen müsste, falls das Wirtschaftswachstum im 21 . Jahrhundert zu Ende geht (oder gehen soll). Prognosen über die Geschwindigkeit zukünftigen Wachstums sind mit großer Unsicherheit behaftet und umstritten (Gordon, 2016). Selbst wenn man ein Ende des Wirtschaftswachstums in reichen Ländern für nicht plausibel hält, so erscheint die Forschungsfrage lohnend, wie mit einer - aus welchen Gründen auch immer - mittelfristig schrumpfenden Wirtschaft umzugehen sei. Dies ist wegen der längeren Frist eine andere Fragestellung als die Überwindung von Rezessionen ${ }^{14}$ und insbesondere auch relevant vor dem Hintergrund ungewollt schrumpfender Wirtschaftsräume, wie Industriestädten. Unter den in diesem Beitrag diskutierten wachstumskritischen Themen stellt dieses womöglich die vergleichbar größte Lücke in der volkswirtschaftlichen Forschung dar. Wachstumskritische Beiträge haben sich dabei in erster Linie auf die Frage konzentriert, ob im gegenwärtigen Wirtschaftssystem „Wachstumszwänge“ vorliegen, insbesondere im Finanzsystem. Strunz et al. (2017) beleuchten verschiedene Forschungsansätze zu Wachstumszwängen und stellen fest, dass es für das Geldsystem bisher keinem Ansatz gelang, solche Zwänge eindeutig zu zeigen oder zu widerlegen. Sie bemerken jedoch, dass weitere mögliche Wachstumszwänge neben der Verschuldung und der Dynamik des Geldsystems selten diskutiert werden. Sowohl die Frage nach möglichen Wachstumszwängen, als auch die Resilienz wohlfahrtsstaatlicher und wirtschaftlicher Systeme gegenüber gewollten oder ungewollten Veränderungen in der Wachstumsrate markieren ein wichtiges Forschungsfeld und könnten wichtige Ansatzpunkte für den konstruktiven Dialog zwischen wachstumskritischen Autoren und wachstumsbefürwortenden Positionen sein.

Drittens steht in manchen wachstumskritischen Ansätzen nicht das richtige Wohlfahrtskonzept im Mittelpunkt, sondern eine wahrgenommene Ausdehnung ökonomischer Logik auf immer mehr Lebensbereiche bzw. die Frage, welche gesellschaftlichen Bereiche marktbasiert organisiert werden sollten ${ }^{15}$ (vgl. Sandel, 2012): So könnte eine Kommerzialisierung und marktbasierte Organisation etwa des Gesundheits- und Bildungswesens und womöglich auch von Teilen der Arbeitswelt schädlich für Ziele wie etwa den sozialen Zusammenhalt, die gesellschaftliche Teilhabe und die „Befähigung zum Spiel“ (Nussbaum, 2011) sein. Bei dieser Auslegung der Wachstumskritik bleibt allerdings fraglich, warum sie spezifisch eine Kritik am Wirtschaftswachstum darstellen soll und nicht stattdessen am Verhältnis zwischen Markt und Staat in der Organisation bestimmter Bereiche des Wirtschaftssystems.

${ }^{14}$ Ein verwandter wachstumskritischer Einwand versteht die Sorge vor einem „Peak Oil“ bzw. „Peak Mineral“ (Cordey et al. 2014) so, dass Institutionen, insbesondere Märkte, womöglich keine verlässlichen Indikatoren für Knappheiten bereitstellen. So wird befürchtet, dass eine von Gesellschaften nicht rechtzeitig erkannte Rohstoffknappheit eine Wirtschaftskrise auslösen könnte. Dieses Argument erscheint gültig, obschon neue volkswirtschaftliche Forschung diese Sorge jedenfalls global eher unbegründet erscheinen lässt (vgl. Stuermer und Schwerhoff, 2013).

${ }^{15}$ Laut Serge Latouche geht es bei einem Wandel hin zu einer Gesellschaft der Décroissance unter anderem „um ein Ent-Ökonomisieren unserer Realität, um eine Aufhebung des fiktiven Warencharakters von Boden, Arbeit und Geld.“ und darum, „die Dominierung der Welt durch die Ökonomie [zu] beenden“ (Latouche in: Seidl und Zahrnt, 2010). 
Darüber hinaus zielt manche Wachstumskritik womöglich auf kapitalistische oder marktwirtschaftliche Systeme im Allgemeinen ab. Manche Stimmen gehen von der Existenz mit dem Kapitalismus untrennbar verbundener Wachstumszwänge aus, die unausweichlich einen wachsenden Ressourcenverbrauch nach sich ziehen. Umweltprobleme wären dann nicht mehr durch die Verbesserung oder Ergänzung einzelner Märkte oder durch Schrumpfen zu bewältigen, sondern nur noch durch einen Systemwechsel. Doch in welchen Systemen könnte Wachstum ausgeschlossen oder Wachstum und Ressourcenverbrauch besser entkoppelt werden? Insofern Wachstum durch grundlegendes menschliches Streben nach Verbesserungen, Innovationen und Kooperation entsteht, wäre es nicht auf marktwirtschaftliche Systeme begrenzt. Historisch sind auch planwirtschaftliche Alternativsysteme überwiegend gewachsen allerdings mit einer noch schlechteren Umweltbilanz als Marktwirtschaften. Die Analyse institutioneller Reformen scheint daher aus der Umweltsicht vielversprechender als eine Systemdiskussion.

\section{Zusammenfassung und Ausblick}

Der vorliegende Beitrag prüft zwei im wachstumskritischen Diskurs verbreitete Argumente auf ihre Stichhaltigkeit. Erstens beruht ein oftmals vorgebrachtes Argument, dass Klimaschutz mit weiterem Wirtschaftswachstum nicht vereinbar sei, auf einem unzulässigen Schluss von einer spezifischen durch politischen Unwillen und institutionelle Hürden gekennzeichneten Klimapolitik in der Vergangenheit auf die Unmöglichkeit technologischer Lösungen und adäquater Regulierung in der Zukunft. Zwar ist es unwahrscheinlich, dass in der Zukunft alle politischen und institutionellen Hürden für globalen optimalen Klimaschutz überwunden werden können. Dennoch scheint für die Durchsetzung von Klimapolitik in einer Zukunft Wirtschaftswachstum eher hilfreich als schädlich, insbesondere was die Entschärfung von Verteilungskonflikten anbelangt, die sich auf dem Weg zur klimaneutralen Wirtschaftsweise ergeben. Dies gilt selbstverständlich nur bei Einhaltung aller überlebensnotwendigen planetaren Grenzen: bei der Steigerung von Wohlergehen durch die Verwirklichung ökologischer und sozialpolitischer Ziele sollte das Erreichen von Wachstum nur eine untergeordnete Rolle spielen. Ob zweitens weiteres Wirtschaftswachstum in reichen Ländern überhaupt erstrebenswert sei, hängt entscheidend vom normativen Standpunkt ab. Wenn Wirtschaftspolitik tatsächlich bestimmte Formen des Glücks in der Bevölkerung steigern soll, so ist zumindest mit herkömmlichem Wachstum des wirtschaftlichen Outputs nur wenig gewonnen. Das heißt aber nicht, dass zusätzlicher Output nicht gezielter als bisher zur Glückssteigerung eingesetzt werden könnte. Die traditionelle ökonomische Bewertung von Wirtschaftspolitik mittels Präferenzerfüllung gibt hingegen wenig Anlass dazu, weiteres Wirtschaftswachstum für gesellschaftlich nicht zielführend zu halten.

Obwohl sich beide Argumente als nicht haltbar erweisen, ergeben sich aus der erfolgten Analyse der wachstumskritischen Argumente einige Folgerungen für volkswirtschaftliche Forschung und deren Kommunikation. Auch hier liefern 
wachstumskritische Stimmen also wichtige Anregungen um den wissenschaftliche Fortschritt zu befördern:

Hinsichtlich der Lösungsansätze für Umweltprobleme scheint ein großer Teil der volkswirtschaftlichen Forschung nicht klar genug zu kommunizieren, dass die Regulierung fast immer im Zweitbesten, also unter Berücksichtigung politischer und institutioneller Hürden, analysiert werden muss. Nur wenn die optimale Regulierung eines Umweltproblems als normativer Maßstab gilt, an dem Politikinstrumente im Zweitbesten insbesondere unter Verteilungskonflikten gemessen werden, lässt sich der Eindruck vermeiden, dass die grundsätzliche Rücknahme von Wachstum dem Umweltschutz dienlich sein könne. Darüber hinaus wird die Möglichkeit der Schrumpfung lediglich spezifischer Sektoren nicht klar genug kommuniziert, beispielsweise was den Flugverkehr anbetrifft.

Was die verwandte Frage einer möglichen Nutzenminderung durch Umweltregulierung angeht, so sind oftmals die Grenzen zwischen positiver und normativer Analyse nicht klar genug gezogen. Dies gilt insbesondere für spezifische Veränderungen der Konsummuster, etwa bezüglich der Ernährung, als notwendigen Beitrag zur Dekarbonisierung. In manchen wirtschaftswissenschaftlichen Beiträgen werden aus "objektiven" Ergebnissen politische Handlungsempfehlungen abgeleitet, die aber auf einer nicht klar formulierten normativen Grundlage beruhen - während eine stattdessen notwendige ethische Analyse als etwas „subjektives“ verstanden wird (Levitt und Dubner, 2009) - und daher keine zwingende Konsequenz empirischer Befunde darstellt (Aldred, 2009). Eine mangelnde Hervorhebung des eingenommenen normativen Standpunkts, sowie die Vernachlässigung alternativer normativer Standpunkte, erschwert jedoch den wissenschaftlichen Diskurs über unterschiedliche gesellschaftliche Ziele, insbesondere darüber, worin „das gute Leben“ für das Individuum bestehe. Dies mag zur Ansicht führen, dass Ökonomen die weit verbreitete Kritik an einer von Konsum geprägten Gesellschaft nicht genug thematisieren.

Zusammenfassend lässt sich festhalten: Es wird völlig zurecht kritisiert, dass manche Bereiche von Politik und Gesellschaft Wirtschaftswachstum zum Selbstzweck erhoben hätten. Dieser Beitrag zeigt jedoch, dass Nicht-Wachstum kein sinnvolleres Alternativziel sein kann. Eine Aufweichung dieser Zweiteilung im gesellschaftlichen Diskurs könnte zu Fortschritt beim Klimaschutz und zu mehr Wohlergehen führen.

\section{Dank}

Die Autoren danken Myles Allen, Eric Beinhocker, Moritz Drupp, Frithjof Gressmann Cameron Hepburn, Philip Mathé, Michael Oberhaus, Johanna Joy Obst, Eugen Pissarskoi, Alexander Radebach, Matthias Roesti, Max Roser, Anselm Schultes, Harry Schindler und Daniel Weishaar für hilfreiche Kommentare und Unterstützung. Linus Mattauch dankt der Sachverständigengruppe „Weltwirtschaft und Sozialethik“ der Deutschen Bischofskonferenz für vielfältige Anregungen; die Autoren danken der Studienstiftung des deutschen Volkes. Linus Mattauchs Forschungsarbeit wurde mit Unterstützung eines Stipendiums im Rahmen des Postdoc-Programms des DAAD ermöglicht. 


\section{Literaturverzeichnis}

Agora Energiewende (2017). Erneuerbare vs. fossile Stromsysteme: ein Kostenvergleich. Bericht der Agora Energiewende basierend auf einer Analyse des Öko-Instituts. Berlin.

Aldred, J. (2009). The Skeptical Economist: Revealing the Ethics Inside Economics. London: Earthscan.

Ariely, D., Loewenstein, G., \& Prelec, D. (2006). Tom Sawyer and the construction of value. Journal of Economic Behavior \& Organization, 60(1), 1-10.

Banister, D. (2008). The sustainable mobility paradigm. Transport policy, 15(2), 73-80.

Baumeister, R. F., Vohs, K. D., Aaker, J. L., und Garbinsky, E. N. (2013). Some key differences between a happy life and a meaningful life. The Journal of Positive Psychology, 8(6), 505516.

Benjamin, D. J., Heffetz, O., Kimball, M. S., und Rees-Jones, A. (2012). What do you think would make you happier? What do you think you would choose? American Economic Review, 102(5), 2083-2110.

Bennholdt-Thomsen, V. (2010). Geld oder Leben: was uns wirklich reich macht. München: OekomVerlag.

Bilancini, E., und D'Alessandro, S. (2012). Long-run welfare under externalities in consumption, leisure, and production: A case for happy degrowth vs. unhappy growth. Ecological Economics, 84, 194-205.

Clarke L., K. Jiang, K. Akimoto, M. Babiker, G. Blanford, K. Fisher-Vanden, J.-C. Hourcade, V. Krey, E. Kriegler, A. Löschel, D. McCollum, S. Paltsev, S. Rose, P.R. Shukla, M. Tavoni, B.C.C. van der Zwaan, und D.P. van Vuuren (2014). Assessing Transformation Pathways. In: Climate Change 2014: Mitigation of Climate Change. Contribution of Working Group III to the Fifth Assessment Report of the Intergovernmental Panel on Climate Change [Edenhofer, O., R. Pichs-Madruga, Y. Sokona, E. Farahani, S. Kadner, K. Seyboth, A. Adler, I. Baum, S. Brunner, P. Eickemeier, B. Kriemann, J. Savolainen, S. Schlömer, C. von Stechow, T. Zwickel and J.C. Minx (Hrsg.)]. Cambridge University Press, Cambridge, United Kingdom und New York, NY, USA.

Creutzig, F. (2016). Economic and ecological views on climate change mitigation with bioenergy and negative emissions. GCB bioenergy, 8(1), 4-10.

Creutzig, F., Jochem, P., Edelenbosch, O. Y., Mattauch, L., van Vuuren, D. P., McCollum, D., \& Minx, J. (2015). Transport: A roadblock to climate change mitigation?. Science, 350(6263), 911-912.

Crisp, R. (2001). In: "Well-Being", The Stanford Encyclopedia of Philosophy (Fall 2017 Edition), Edward N. Zalta (ed.), URL = https://plato.stanford.edu/archives/fall2017/entries/wellbeing/, abgerufen: 14.2.2019.

Deutscher Bundestag (2013). Schlussbericht der Enquete-Kommission ,Wachstum, Wohlstand, Lebensqualität-Wege zu nachhaltigem Wirtschaften und gesellschaftlichem Fortschritt in der Sozialen Marktwirtschaft “. Drucksache, 17/13300.

Dietz, S., Bowen, A., Doda, B., Gambhir, A., Warren, R. (2018). The economics of 1.5 C climate change. Annual Review of Environment and Resources, 43, 455-480.

Easterlin, R. A., McVey, L. A., Switek, M., Sawangfa, O., und Zweig, J. S. (2010). The happinessincome paradox revisited. Proceedings of the National Academy of Sciences, 107(52), 22463-22468.

Europäische Kommission (2017). Emission Database for Global Atmospheric Research (EDGAR), release version 4.2. European Commission, Joint Research Centre (JRC)/PBL Netherlands Environmental Assessment Agency. http://edgar.jrc.ec.europa.eu, (abgerufen am 12.9.2017).

Edenhofer, O., Flachsland, C., und Brunner, S. (2011). Wer besitzt die Atmosphäre?. Leviathan, 
39(2), 201-221.

Ellenbeck, S. (2017). Modelle in der Klimaökonomik: Instrument, Bild oder Dispositiv? Eine wissenssoziologische Annäherung. Leviathan, 45(1), 111-130.

Fleurbaey, M., und Blanchet, D. (2013). Beyond GDP: Measuring Welfare and Assessing Sustainability. Oxford University Press.

Fuss, S., Canadell, J. G., Peters, G. P., Tavoni, M., Andrew, R. M., Ciais, P., ... \& Le Quéré, C. (2014). Betting on negative emissions. Nature Climate Change, 4(10), 850-853.

Georgescu-Roegen, N. (1971). The Entropy Law and the Economic Process. Harvard University Press, Cambridge, Mass.

Georgescu-Roegen, N. (1986). The Entropy Law and the Economic Process in Retrospect. Eastern Economic Journal, 12(1), 3-25.

Gillingham, K., und Palmer, K. (2014). Bridging the energy efficiency gap: Policy insights from economic theory and empirical evidence. Review of Environmental Economics and Policy, 8(1), 18-38.

Gordon, R. J. 2016. Perspectives on The Rise and Fall of American Growth. American Economic Review, 106(5), 72-76.

Greene, J. D. (2013). Moral Tribes: Emotion, Reason, and the Gap Between Us and Them. London: Penguin.

Gintis, H. (1974). Welfare criteria with endogenous preferences: the economics of education. International Economic Review, 415-430.

Guivarch, C., \& Hallegatte, S. (2011). Existing infrastructure and the $2 \mathrm{C}$ target. Climatic Change, 109(3-4), 801-805.

Hausman, D. M. (2012). Preference, value, choice, and welfare. Cambridge: Cambridge University Press.

Heinberg, R. (2007). Peak everything: waking up to the century of declines. New Society Publishers.

Helm, D. (2010). Government failure, rent-seeking, and capture: the design of climate change policy. Oxford Review of Economic Policy, 26(2), 182-196.

Hepburn, C., und Bowen, A. (2013). Prosperity with growth: Economic growth, climate change and environmental limits. In R. Fouquet (Ed.), Handbook of Energy and Climate Change (pp. 617-638). Cheltenham: Edward Elgar.

Hsee, C. K., und Hastie, R. (2006). Decision and experience: why don't we choose what makes us happy? Trends in Cognitive Sciences, 10(1), 31-37.

IEA (2016). Recent trends in the OECD: Energy and $\mathrm{CO}_{2}$ emissions (2016 preliminary). International Energy Agency. https://www.iea.org/media/statistics/Recent_Trends_in_the_OECD.pdf, (abgerufen am 06.09.2017).

IPCC (2014). Climate Change 2014: Mitigation of Climate Change. Contribution of Working Group III to the Fifth Assessment Report of the Intergovernmental Panel on Climate Change. [Edenhofer, O., R. Pichs-Madruga, Y. Sokona, E. Farahani, S. Kadner, K. Seyboth, A. Adler, I. Baum, S. Brunner, P. Eickemeier, B. Kriemann, J. Savolainen, S. Schlömer, C. von Stechow, T. Zwickel und J.C. Minx (Hrsg.)]. Cambridge University Press, Cambridge, United Kingdom und New York, NY, USA.

IPCC Technical Summary (2014). Technical Summary. Edenhofer O., R. Pichs-Madruga, Y. Sokona, S. Kadner, J. C. Minx, S. Brunner, S. Agrawala, G. Baiocchi, I. A. Bashmakov, G. Blanco, J. Broome, T. Bruckner, M. Bustamante, L. Clarke, M. Conte Grand, F. Creutzig, X. Cruz-Núñez, S. Dhakal, N. K. Dubash, P. Eickemeier, E. Farahani, M. Fischedick, M. Fleurbaey, R. Gerlagh, L. Gómez-Echeverri, S. Gupta, J. Harnisch, K. Jiang, F. Jotzo, S. Kartha, S. Klasen, C. Kolstad, V. Krey, H. Kunreuther, O. Lucon, O. Masera, Y. Mulugetta, R. B. Norgaard, A Patt, N. H. Ravindranath, K. Riahi, J. Roy, A. Sagar, R. Schaeffer, S. Schlömer, K. C. Seto, K. Seyboth, R. Sims, P. Smith, E. Somanathan, R. 
Stavins, C. von Stechow, T. Sterner, T. Sugiyama, S. Suh, D. Ürge-Vorsatz, K. Urama, A. Venables, D. G. Victor, E.Weber, D. Zhou, J. Zou, and T. Zwickel. In: Climate Change 2014: Mitigation of Climate Change. Contribution of Working Group III to the Fifth Assessment Report of the Intergovernmental Panel on Climate Change [Edenhofer, O., R. Pichs-Madruga, Y. Sokona, E. Farahani, S. Kadner, K. Seyboth, A. Adler, I. Baum, S. Brunner, P. Eickemeier, B. Kriemann, J. Savolainen, S. Schlömer, C. von Stechow, T. Zwickel and J. C. Minx (Hrsg.)]. Cambridge University Press, Cambridge, United Kingdom and New York, NY, USA.

IPCC (2018). Global warming of $1.5^{\circ}$ C. World Meteorological Organization.

Jackson, T. (2017). Prosperity without Growth: Foundations for the Economy of Tomorrow. London: Routledge.

Jakob, M., und Edenhofer, O. (2014). Green growth, degrowth, and the commons. Oxford Review of Economic Policy, 30(3), 447-468.

Jakob, M. und Hilaire, J. (2015). Climate science: Unburnable fossil-fuel reserves. Nature 517: $150-152$

Kahneman, D., und Deaton, A. (2010). High income improves evaluation of life but not emotional well-being. Proceedings of the National Academy of Sciences of the United States of America, 107(38), 16489-16493.

Kahneman, D., und Krueger, A. B. (2006). Developments in the measurement of subjective wellbeing. The Journal of Economic Perspectives, 20(1), 3-24.

Kahneman, D., Krueger, A. B., Schkade, D. A., Schwarz, N., und Stone, A. A. (2004). A survey method for characterizing daily life experience: The day reconstruction method. Science, 306(5702), 1776-1780.

Kallis, G. (2011). In defence of degrowth. Ecological Economics, 70(5), 873-880.

Klenert, D., Mattauch, L., Combet, E., Edenhofer, O., Hepburn, C., Rafaty, R. and Stern, N. (2018) Making carbon pricing work for citizens. Nature Climate Change, 8: 669-677.

Lancaster, K. J. (1966). A new approach to consumer theory. Journal of Political Economy, 74(2), 132-157.

Latouche, S. (2015). Es reicht! Abrechnung mit dem Wachstumswahn. München: Oekom-Verlag.

Layard, R. (2011). Happiness: Lessons from a new science. London: Penguin UK.

Leipprand, A., und aus dem Moore, N. (2012). Die natürlichen Grenzen der Ökonomie: Plädoyer für eine ökologische Schuldenbremse. Leviathan, 40(2), 173-201.

Levitt, S. D., und Dubner, S. J. (2009). Freakonomics: A Rogue Economist Explores the Hidden Side of Everything. New York: HarperCollins.

Lipsey, R. G., und Lancaster, K. (1956). The General Theory of Second Best. The Review of Economic Studies, 24(1), 11-32.

Loewenstein, G., und Ubel, P. A. (2008). Hedonic adaptation and the role of decision and experience utility in public policy. Journal of Public Economics, 92(8), 1795-1810.

Louwen, A., van Sark, W., Faaij, A. und Schropp, R. (2016). Re-assessment of net energy production and greenhouse gas emissions avoidance after 40 years of photovoltaics development. Nature Communications, Vol. 7, 13728.

Loske, $R$. (2014). Neue Formen kooperativen Wirtschaftens als Beitrag zur nachhaltigen Entwicklung-Überlegungen zur Wiedereinbettung der Ökonomie in Gesellschaft und Natur. Leviathan, 42(3), 463-485.

Malthus, T. (1798). An essay on the principle of population. London: J. Johnson.

Mattauch, L., und Hepburn, C. (2016). Climate Policy When Preferences Are Endogenous - And Sometimes They Are. Midwest Studies In Philosophy, 40, 76-95.

Mattauch, L., Ridgway, M., \& Creutzig, F. (2016). Happy or liberal? Making sense of behavior in transport policy design. Transportation research part D: transport and environment, 45, 64-83.

Meadows, D. ., Meadows, D., Randers, J., und Behrens, W. (1972). The Limits to Growth. A 
Report for the Club of Rome's Project on the Predicament of Mankind. Washington D.C.: Potpmac Associates.

Miegel, M. (2010). Wohlstand ohne Wachstum. Berlin: Propyläen Verlag.

Northey, S., Mohr, S., Mudd, G. M., Weng, Z., \& Giurco, D. (2014). Modelling future copper ore grade decline based on a detailed assessment of copper resources and mining. Resources, Conservation and Recycling, 83, 190-201.

Nussbaum, M. (2011). Creating capabilities. Cambridge (MA): Harvard University Press.

Nussbaum, M. (2001). Women and Human Development: The Capabilities Approach. Cambridge: Cambridge University Press.

Paech, N. (2009). Die Postwachstumsökonomie-ein Vademecum. Zeitschrift Für Sozialökonomie, 46(160-161), 28-31.

Paech, N. (2013). Befreiung vom Uberfluss. Auf dem Weg in die Postwachstumsökonomie (5. Auflage). München: Oekom-Verlag.

Perman, R., Ma, Y., McGilvray, J., und Common, M. (2003). Natural resource and environmental economics (3. Auflage). Harlow: Pearson Education.

Pissarskoi, E. (2016). Integrated Assessment Models - Kompass in einem magnetisierten Umfeld? Ökologisches Wirtschaften, 4 (31), 39-44.

Pindyck, R. S. (2013). Climate change policy: what do the models tell us?. Journal of Economic Literature, 51(3), 860-72.

Raworth, K. (2017). Doughnut Economics: Seven Ways to Think Like a 21st-Century Economist. Chealsea, Vermont: Chelsea Green Publishing.

Sacks, D. W., Stevenson, B., und Wolfers, J. (2012). The New Stylized Facts about Income and Subjective Well-Being. Emotion, 12(6), 1181-1187.

Sandel, M. J. (2012). What money can't buy: the moral limits of markets. New York: Macmillan.

Sandén, B. und Arvesen, A. (2015). Energy balance and climate impact of renewable power: is there cause for concern? In: Sandén, A. (ed.), Systems Perspectives on Renewable Power 2014. Chalmers University of Technology, Göteborg.

Schmelzer, M., und Passadakis, A. (2011). Postwachstum. Krise, ökologische Grenzen und soziale Rechte. Hamburg: VSA-Verlag.

Schmelzer, M. (2015). Spielarten der Wachstumskritik. In Atlas der Globalisierung (S. 116-121). Berlin: taz Verlags-und Vertriebs GmbH.

Scitovsky, T. (1976). The Joyless economy. Oxford: Oxford University Press.

Seidl, I., und Zahrnt, A. (Hrsg.). (2010). Postwachstumsgesellschaft: Konzepte für die Zukunft. Weimar (Lahn): Metropolis-Verlag.

Sen, A. (1985). Commodities and Capabilities. Amsterdam: North-Holland.

Siegmeier, J., Mattauch, L., und Edenhofer, O. (2018). Capital beats coal. How collecting the climate rent increases aggregate investment. Journal of Environmental Economics and Management, 88, 366-378.

Sims R., R. Schaeffer, F. Creutzig, X. Cruz-Núñez, M. D'Agosto, D. Dimitriu, M. J. Figueroa Meza, L. Fulton, S. Kobayashi, O. Lah, A. McKinnon, P. Newman, M. Ouyang, J. J. Schauer, D. Sperling, and G. Tiwari (2014): Transport. In: Climate Change 2014: Mitigation of Climate Change. Contribution of Working Group III to the Fifth Assessment Report of the Intergovern-mental Panel on Climate Change [Edenhofer, O., R. PichsMadruga, Y. Sokona, E. Farahani, S. Kadner, K. Seyboth, A. Adler, I. Baum, S. Brunner, P. Eickemeier, B. Kriemann, J. Savolainen, S. Schlömer, C. von Stechow, T. Zwickel and J.C. Minx (eds.)]. Cambridge University Press, Cambridge, United Kingdom and New York, NY, US.

Steffen, W., Richardson, K., Rockström, J., Cornell, S. E., Fetzer, I., Bennett, E. M., Biggs, R., Carpenter, S.R., de Vries, W., de Wit, C.A., Folke, C., Gerten, D., Heinke, J., Mace, G.M., Persson, L.M..,Ramanathan, V., Reyers, B., Sörlin, S. (2015). Planetary boundaries: Guiding human development on a changing planet. Science, 347(6223), 1259855. 
Stuermer, M.,und Schwerhoff, G. (2013). Technological change in resource extraction and endogenous growth. Bonn Econ Discussion Paper 12/2013.

Stiglitz, J., Sen, A., und Fitoussi, J.-P. (2009). The measurement of economic performance and social progress revisited. Reflections and Overview. Commission on the Measurement of Economic Performance and Social Progress, Paris.

Stiglitz, J. E., Stern, N., Duan, M., Edenhofer, O., Giraud, G., Heal, G., Lèbre la Rovere, E., Morris, A., Moyer, E., Pangestu, M., Shukla, P.R., Sokona, Y. und Winkler, H. (2017). Report of the high-level commission on carbon prices. Carbon Pricing Leadership Coalition. 29. Mai 2017.

Strunz, S., Bartkowski, B. und Schindlerm H. (2017). Is there a monetary growth imperative? In Victor, P., Dolter, B., Handbook on Growth and Sustainability (S.326-256), Cheltenham: Edward Elgar.

UBA (2019). Treibhausgasemissionen 2017 leicht gesunken. Umweltbundesamt, Pressemitteilung 03/2019 vom 18.1.2019.

https://www.umweltbundesamt.de/presse/pressemitteilungen/treibhausgasemissionen2017-leicht-gesunken (abgerufen am 22.2.2019).

van den Bergh, J. C. J. M. (2017). A third option for climate policy within potential limits to growth. Nature Climate Change, 7(2), 107-112.

Weyant, J. (2017); Some Contributions of Integrated Assessment Models of Global Climate Change, Review of Environmental Economics and Policy, 11(1), 115-137. 